Review

\title{
The Application of Patient-Derived Xenograft Models in Gynecologic Cancers
}

\author{
Wenxiao Jiang, Shangdan Xie, Yi Liu, Shuangwei Zou, Xueqiong Zhu ${ }^{\bowtie}$ \\ Department of Obstetrics and Gynecology, the Second Affiliated Hospital of Wenzhou Medical University, Wenzhou, Zhejiang, 325027, China. \\ $\triangle$ Corresponding author: Xueqiong Zhu, Address: No. 109 Xueyuan Xi Road, Department of Obstetrics and Gynecology, the Second Affiliated Hospital of \\ Wenzhou Medical University, Wenzhou, Zhejiang, 325027, China. Tel: +86 577 88002796; Fax: +86 577 88832693; E-mail: Xueqiong Zhu, zjwzzxq@163.com. \\ (1) The author(s). This is an open access article distributed under the terms of the Creative Commons Attribution License (https://creativecommons.org/licenses/by/4.0/).
} See http://ivyspring.com/terms for full terms and conditions.

Received: 2020.03.19; Accepted: 2020.06.14; Published: 2020.07.11

\begin{abstract}
Recently, due to the limitations of cell line models and animal models in the preclinical research with insufficient reflecting the physiological situation of humans, patient-derived xenograft (PDX) models of many cancers have been widely developed because of their better representation of the tumor heterogeneity and tumor microenvironment with retention of the cellular complexity, cytogenetics, and stromal architecture. PDX models now have been identified as a powerful tool for determining cancer characteristics, developing new treatment, and predicting drug efficacy. An increase in attempts to generate PDX models in gynecologic cancers has emerged in recent years to understand tumorigenesis. Hence, this review summarized the generation of PDX models and engraftment success of PDX models in gynecologic cancers. Furthermore, we illustrated the similarity between PDX model and original tumor, and described preclinical utilization of PDX models in gynecologic cancers. It would help supply better personalized therapy for gynecologic cancer patients.
\end{abstract}

Key words: patient-derived xenograft models; gynecologic cancers; characteristics; application; preclinical utilization

\section{Introduction}

Nowadays, more than 113,520 new gynecologic cancer cases were diagnosed and almost 33,620 mortalities have occurred in the United States [1]. Gynecologic cancers include ovarian cancer, cervical cancer, endometrial/uterine cancer, vulvar cancer, and vaginal cancer, according to tumor locations [2]. Although treatment strategies have been developed in recent years, the survival rate has not notably improved and many patients still undergo cancer relapse due to the highly heterogeneity in tumors. For example, in recent cancer statistics, five-year relative survival rate for females with ovarian cancer and cervical cancer were reported only about $48 \%$ and $66 \%$, respectively [1]. Cervical cancer is one of the most common malignancies in females worldwide with a poor overall prognosis for metastatic or recurrent cases [3]. Currently, patients with recurrent or metastatic cervical cancer are treated with chemotherapy, but their responses to single and combination therapies still remain poor. Ovarian cancer is a highly malignant cancer and the most deadly cancer in gynecology [4], with almost $75 \%$ cases developing relapse or chemoresistance after initial response to platinum-based therapies. Chemoresistance and recurrence are the most leading causes of death especially in women with high-grade serous ovarian cancer (HGS-OC). Hence, it is urgent to seek for personal and precise therapeutic targets for gynecologic cancers especially ovarian and cervical cancers.

To our knowledge, heterogeneity in gynecologic malignancies contains histopathology, inter-cancer and population heterogeneity, which makes it difficult to obtain a cure with current therapies. Emerging evidence has confirmed the importance of personalized treatment approaches targeting molecular alterations for individual patients. Preclinical researches in gynecologic cancers largely 
rely on cloned cancer-derived cell lines, cell linesderived tumor xenografts and animal models. Regretfully, animal models such as mice are so extremely different from humans [5] and insufficiently reflect the physiological situation in humans, subsequently leading to treatment failures in clinical trials [6]. Conventional cell lines that are cultured in vitro and in vivo lose their original tumor characteristics due to genetic and phenotypic alterations when transplanted [7]. As a result, patient-derived xenograft (PDX) model has recently been established to overcome these disadvantages, and become the most reliable human cancer model in vivo for preclinical research, as it accurately recapitulates molecular, genetic, histological, and chemo-responsive characteristics of original cancer [8-11], improving therapeutic strategies against gynecologic cancers. PDX model has been largely applied to the researches of cancer drug resistance [12] and molecular mechanism of relapsed and metastatic tumors [13,14], assessment of anti-tumor drug efficacy and discovery of new anti-cancer medicines [15].

Currently, more and more evidence has witnessed the application of PDX models in numerous gynecologic cancers including ovarian cancer $[16,17]$, and cervical cancer $[18,19]$, improving our understanding of cancer biology and mechanisms of therapeutic response in gynecologic cancers. Hence, this review is designed to assess the application and current preclinical utilization of PDX models in the field of gynecologic cancer, for providing more opportunities to optimize these models to develop clinical guidelines to manage gynecologic cancer treatment.

\section{Generation of PDX models}

PDX models are acquired by direct engraftment of patient biopsy or surgical dissected tumor tissues into immuno-deficient mice and subsequent transplantation into passage recipient mice (Figure 1). Generally, these models are performed through heterotopic or orthotopic implantation. Unlike orthotopic injection, heterotopic implantation occurs when cancer samples are injected into a mouse site independent on the primary cancer location, generally subcutaneously, by sub-renal capsular, in the interescapular region, or through the mammary fat pad $[20,21]$. Most popular models currently offered to patients are subcutaneous-transplantation in immuno-deficient mice, which rarely metastasize and uncommonly simulate the initial tumor microenvironment [22]. Subrenal capsule grafting can largely improve tumor engraftment success and reservation of human cancer heterogeneity [23]. In contrast, orthotopic-transplant PDX models can generate metastasis and accurately mimic the natural environment of primary tumor, which are usually used for the study of tumor metastasis [24]. For most ovarian cancers, research is frequently performed using heterotopically transplanted PDX models, because it is technically easy and can monitor cancer size accurately.

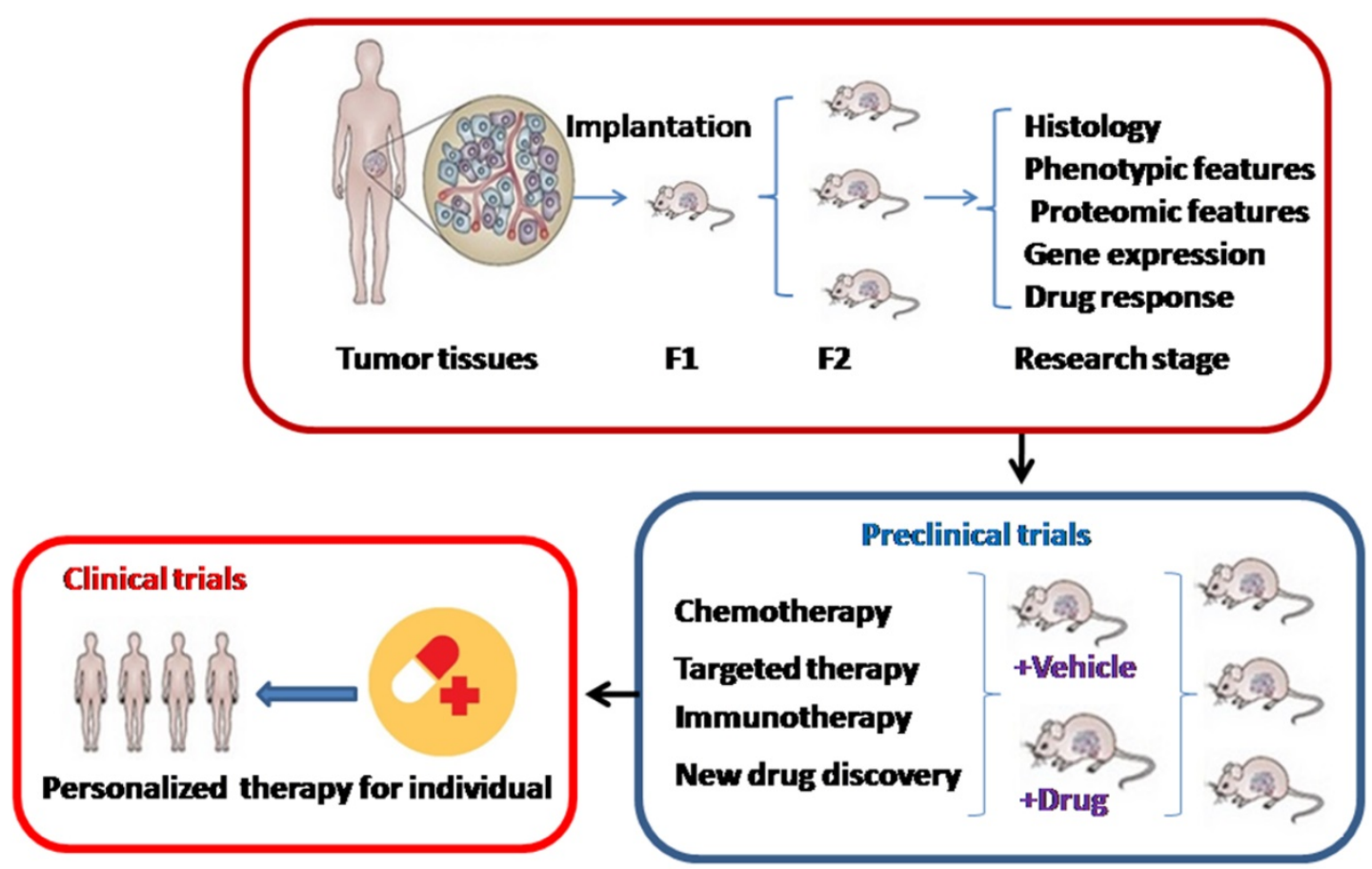

Figure 1. The development and application of patient-derived xenograft (PDX) models. F1: Cancer tissues are engrafted directly into immuno-deficient mice. F2: Then cancers are transplanted into a second generation of immuno-deficient mice. 
In addition, the most common mouse strains include severe combined immuno-deficient (SCID), non-obese diabetic (NOD)/severe combined immuno-deficient (SCID), NOD/SCID/IL2Ry null (NSG), and athymic nude mice [25]. In gynecologic cancers especially ovarian cancer, NSG and SCID mice are the most frequently used hosts due to their high engraftment rate $[26,27]$. Sometimes nude mice, lacking thymus and $\mathrm{T}$ lymphocytes, are also used for gynecologic tumor xenografts because of its cheap expend. Moreover, the time to tumor formation is varied among cancers. For example, the establishment time in high-risk endometrial cancer PDXs was between 2 and 11 weeks [28]. It was shorter than the length of tumor establishment in cervical cancer PDX models, whose mean length of time was $32.4+/-3.5$ weeks and similar to the time in its successive transplantations [18].

Furthermore, it is also important to provide a useful imaging tool for monitoring of PDX cancer models in gynecologic cancers. Apparent diffusion coefficient (ADC) values derived from diffusionweighted magnetic resonance imaging (DW-MRI) could reflect the cellular environment of biological tissues. In four cervical squamous cell carcinoma PDX models, one group observed that median tumor ADC was negatively related to the fraction of collagen I, suggesting that DW-MRI may be a non-invasive imaging approach for characterizing the stromal microenvironment of cervical cancer [29]. Consistently, four cervical cancer PDX models were used to detect the correlation between dynamic contrast-enhanced (DCE) MRI and parameters of the tumor microenvironment, and it was noticed that DCE-MRI provided valuable information on the hypoxic fraction of cervical squamous cell carcinoma [30]. In the study of endometrial carcinoma PDX models, Haldorsen et al. [31] described that the positron emission tomography (PET) tracers imaging methods found metastasis at $82 \%(9 / 11)$ of the necropsy mice, suggesting 18F-fluorodeoxyglocose (18F-FDG) is a promising imaging tool for monitoring PDX models in endometrial cancer.

\section{Engraftment success of PDX models in gynecologic cancers}

Engraftment rate is often influenced by multiple factors, including the characteristics of cancer subtypes, host strain, implantation site, primary versus metastatic tumors, patient's treatment status, and preservation of the tumor specimens.

\section{Cancer subtypes}

The engraftment rate is various according to different gynecologic cancers and different tumor types. The engraftment success rate of cervical cancer PDX models was reported from $66.7 \%$ to $71.4 \%$ $[18,19]$, while the PDXs engraftment rate of endometrial cancer was between $60 \%$ and $86 \%[32,33]$. As to ovarian cancer PDX models, engraftment success ranged from $45.5 \%$ to $100 \%$ [34-36]. Among uterine sarcomas, a kind of rare and heterogeneous gynecologic tumor, it was identified that the engraftment rate of leiomyosarcomas was $77 \%$ (10/13), compared with $29 \%$ in carcinosarcomas [37].

The engraftment rate also appears to vary depending on cancer subtypes. For instance, the successful engraftment rate of epithelial ovarian cancer (EOC) PDXs ranged from $45.5 \%$ to $48.8 \%$ $[34,38]$, which was similar to the engraftment rate of estrogen receptor (ER)-positive relapsed high grade ovarian cancer (HG-OC) PDXs reaching 52.9\% [39]. Nevertheless, the xenografting success rate of HGSOC PDX models was up to $83 \%$ [40]. In a recent study, Wu et al. [16] uncovered that the tumor formation rate in ovarian carcinoma PDX models was $18.52 \%$, among which the tumor formation rate of nonepithelial ovarian tumor PDXs was higher than that of epithelial ovarian tumor (17.39\%). Meanwhile, they discovered $100 \%$ of tumor formation rate for ovarian germ cell tumor PDX models and 33.33\% for metastatic ovarian cancer PDXs. It may be suggested that a more malignancy cancer presents a higher engraftment rate.

\section{The strain of host}

Success rate differs among various host strains. As early as 1993, it was uncovered a more successful engraftment of human tumor PDXs in SCID mice than that in nude mice [41]. Recently, a systematic review confirmed that the PDX engraftment success in different mice was nude $<\mathrm{SCID}<\mathrm{NOD} / \mathrm{SCID}<\mathrm{NSG}$, suggesting that more immune compromised mice contribute to a more successful PDX engraftment [42].

Regarding ovarian cancer PDX models, better engraftment efficiency was achieved by implanting cancer samples into SICD mice than into BALB/c nude mice or NSG mice [40]. It was noticed that the engraftment rate of ovarian cancer in female CB17 SCID mice was 68\% [43], more successful than $45.5-48.8 \%$ in nude mice $[34,38]$. Additionally, the engraftment success rate of HGS-OC PDXs with subcutaneous and intra-ovarian bursal implantation into NSG mice was $83 \%$ [40], higher than $52.9 \%$ in ER-positive relapsed HGS-OC patients with intraperitoneal implantation into female SCID beige mice [39]. Consistently, the engraftment success rate of cervical cancer PDX models was identified as 71.4 $\pm 12.5 \%$ in NSG mice [18], which was more successful compared with $66.7 \%$ in BALB/c nude mice with subrenal capsule implantation [19]. However, an 
additional recent study addressed that animal species, the initiation site of tumor, cancer malignancy degree, cancer stage, cancer type were not related to the tumor formation rate of ovarian carcinoma [16].

\section{Implantation site}

The implantation site is also an important factor affecting engraftment success. The high-risk endometrial cancer engraftment rate in subrenal capsule models was $62.5 \%$, higher than that in subcutaneous models (50\%) [28]. In EOC PDX models, Colombo et al. [34] implanted cancer tissues into the inter-scapular fat pad of Swiss-nude mice and reported the engraftment success rate was $45.5 \%$, which was slightly lower than $48.8 \%$ when EOC specimen was transplanted into subrenal capsule of female BALB/C-nude mice [38].

\section{Primary versus metastatic tumor}

Another important consideration regarding engraftment rate is metastatic tumor. It was noticed that the engraftment success rate in metastatic or recurrent endometrial cancer PDX models was $60 \%$ [32], which was lower than $77.8 \%$ in high-risk endometrial cancer PDXs including 10 high-grade endometrial cancer models, six serous carcinoma models, one clear cell carcinoma model, and one carcinosarcoma model [28].

\section{The status of patients}

Patients' status also influences the engraftment efficiency. A research delineated that the engraftment success in EOC PDX models was negatively related to the overall survival rate in patients whose tumors were implanted into mice [38], suggesting an inverse correlation between engraftment rate and patients' status. This similar phenomenon was also discovered in other cancers such as lung cancer, showing that high stages (stage III or stage IV), adenocarcinoma and moderately differentiation were related to the PDX engraftment success [44].

\section{Preservation of the tumor specimens}

Considering the effect of the preservation method of tumor specimens to the engraftment success, Alkema et al. [43] created 45 subcutaneous ovarian cancer PDX models from 66 ovarian cancer women in advanced stage (III/IV) and found that the fetal calf serum (FCS)/DMSO-based cryopreservation of ovarian cancer tissues presented a higher take rate of $94 \%$ in comparison with vitrification cryopreservation (67\%) and using fresh PDX cancer samples $(91 \%)$, despite of the overall take rate of $68 \%$.

\section{Other factors}

Of note, it was reported that receptor tyrosine kinase-like orphan receptor (ROR1)-positive cells from ovarian cancer PDXs with a high expression of aldehyde dehydrogenase 1 (ALDH1) were more likely to engraft tumor into immune-deficient mice than ROR1-negative ovarian cancer cells, suggesting that ROR1 expression is associated with the engraft success [45]. Considering the contribution of mouse pain and stress to engraftment success, ovarian cancer PDX models established orthotopically showed that tumor engraftment rate in analgesia-treated groups was similar to that in control group [26].

\section{Similarity between PDX model and original tumor in gynecologic cancers}

Numerous researches have contributed to the study of concordance between PDX models and primary tumors in phenotypic and proteomic characteristics, gene expression, and drug response.

\section{Phenotypic and proteomic features}

Some papers have confirmed a high resemblance in histology, phenotypic features and molecular markers between gynecologic cancer PDX models and donor tumors including ovarian cancer, cervical cancer, endometrial cancer and leiomyosarcomas $[18,19,32,34,37]$. For instance, established orthotopic HGS-OC PDX models in NSG mice from three donors have been found to maintain their original morphology and molecular marker profile [46]. In addition, another research group validated the ability of EOC PDX models to conserve their original phenotypic features and differentiation level, as well as ability to recapitulate the intratumoral heterogeneity of primary cancer [34]. Similarly, cervical cancer PDX models have been validated to have highly retention of morphological, histoarchitecture and immunohistochemical features, including similar p16 INK4a and HPV of the original cancers [18].

Proteomic features of these PDX models have been compared with those of original tumors, which is critical for evaluating the therapeutic trial designs and drug response. Using immunohistochemistry, $\mathrm{Wu}$ et al. [16] addressed that ovarian cancer PDXs expressed some similar proteins to original cancer such as nervous tissue marker (Syn), epithelial tissue marker (CK7), interstitial tissue marker (Vimentin), tumor protein p53, proliferating cell nuclear antigen, proliferative marker antigen Ki-67, and nuclear factor erythroid 2 like 2 (Nrf2). Earlier evidence also confirmed the positive expression of WT1, PAX8, ER, progesterone receptor (PR), as well as $\mathrm{Ki}-67$ in HGS-OC PDXs similar to those in corresponding tumor [40]. It was revealed that desmin and $\mathrm{H}$-caldesmon proteins were expressed in 8 models 
among 10 leiomyosarcomas PDX models, and the changes in vimentin and cytokeratin expression in carcinosarcomas PDX models varied over the generations [37]. Another related evidence revealed that thrombospondin-1 (TSP-1), regulating cell interaction with the microenvironment, was expressed in 10/11 ovarian cancer PDXs and upregulated in the homologous primary tumor, implying similar microenvironment of the PDX and original tumor [47]. On the other hand, it is also critical to illustrate the nonhuman component of expression data in PDX models. Liu et al. [48] described several differential protein kinases in ovarian cancer PDXs compared with donor tumor, such as PDGFRA, PDGFRB and CSF1R.

\section{Gene expression}

With the analysis of transcriptome sequencing, one study identified a similarity in gene expression, gene fusion, gene splicing and single nucleotide polymorphisms between ovarian cancer PDX models and their corresponding tumors, and especially observed a consistent rate of $87.19 \%$ in gene expression with 19,493 co-expressed genes [16]. In the investigation of mRNA differences between nine ovarian cancer PDX models and donor tumors, another research group accounted for $78.4 \%$ gene reads in PDX tumor mapping to the human reference genome, higher than $16.3 \%$ mapping to the host mice genome, and also identified 1,935 PDX-donor differential genes, which enriched in stroma-specific functions. They further deleted these differential genes and found a stronger transcriptional similarity between ovarian cancer PDXs and original tumors with average correlation coefficient increasing from 0.91 to 0.95 [48]. Similarly, EOC PDX models were also demonstrated to reserve the copy number change characteristics of the primary cancer over several passages and maintain an oligoclonal structure, implying that ovarian cancer PDX models reserve at least part of the clonal diversity of the original tumor [34]. In cervical cancer, it was demonstrated that all PDX tumors including serially passage models maintained the genomic DNA alterations observed in original cancers [19]. These suggest that PDX models of gynecologic cancers displayed a high concordance with original tumors in gene expression and the differential expressed genes were mainly related to stroma functions.

In analyzing the gene mutation of ovarian cancer, one research group established HGS-OC PDXs and reported a similar copy number variation to original tumor, and that the frequency of TP53 alterations in PDXs was 93\%, BRCA1 mutation was $13 \%$, BRCA2 mutation was $8 \%$, consistent with the analysis in The Cancer Genome Atlas (TCGA) data [49]. Similarly, another study indicated that the rate of TP53 mutations in ten HGS-OC PDXs was $100 \%$, BRCA1 mutation with methylation was $20 \%$, and BRCA2 mutation was 30\% [40]. A good resemblance in genetic mutation profiles and gene expression was also demonstrated in high-grade endometrial cancer PDX models compared with primary tumors [28]. While, using whole-genome low-coverage sequencing, another group documented that the similarity in copy number profiles between leiomyosarcomas PDX models and donor tumors ranged from $57.7 \%$ to $98.2 \%$ and between carcinosarcomas PDX models and donor tumors was between $47.4 \%$ and $65.8 \%$ [37].

\section{Drug response}

Considering chemotherapy response, a highly concordance of cisplatin and carboplatin sensitivity between HGS-OC PDX models and donor patients was detected [40,49]. In one study, Oh et al. [19] established 14 cervical cancer PDX models and confirmed a high expression of human epidermal growth factor receptor-2 (HER2) in serially passaged PDX models similar to that in original cancer, implying that PDX models can be used to study the response to HER2 target therapy in cervical cancer patients.

\section{Preclinical utilization of PDX models in gynecologic cancers}

PDX models have been used to explore tumor mechanism, identify candidate drugs and monitor therapeutic response in gynecologic cancers for decades.

\section{Standard chemotherapeutics in PDX models of gynecologic cancers}

Drug resistance is one of the major contributing factors that determine the efficacy of chemotherapy in patients. In order to determine gynecologic cancer sensitivity or resistance to standard chemotherapy, an increasing number of researches on PDXs have been carried out. In order to identify the chemo-resistant patients in ovarian cancer, Dobbin et al. [50] compared six pairs of ovarian cancer PDXs which were divided into two groups of combined carboplatin/paclitaxel treatment and no treatment, showing a consistent disparity in genetic profile after therapy, suggesting that heterogeneity of PDX models can be used to identify the chemo-resistant population in ovarian cancer. Similarly, by creating HGS-OC PDX models, paclitaxel/carboplatin standard chemotherapy was demonstrated to markedly reduce PDX tumor weight in comparison with the phosphate 
buffered saline group with statistical significance [38]. Furthermore, a study in ovarian cancer PDX models validated that combination of ifosfamide and paclitaxel was more favorable in inhibiting tumor growth than the treatment of combined carboplatin and paclitaxel [51]. Interestingly, in order to overcome chemo-resistance to paclitaxel, Byeon et al. [52] developed a hyaluronic acid-labeled poly (d,1-lactideco-glycolide) nanoparticle (HA-PLGA-NP) incorporating paclitaxel and focal adhesion kinase (FAK) siRNA and demonstrated that this compound dramatically suppressed cancer growth and overcame chemo-resistance in comparison with paclitaxel alone in a drug-resistant EOC PDX model. Exportin-1 (XPO1) is a nuclear exporter, which mediates nuclear export of various cancer inhibitors. Another study developed platinum-resistant ovarian cancer PDX models and revealed that a XPO1 inhibitor selinexor significantly reduced model tumor growth and benefited mice survival no matter monotherapy or in combination with platinum [53]. Furthermore, it has been known that pregnancy-associated plasma protein-A (PAPP-A) suppression is associated with platinum sensitivity in chemotherapy. Torres et al. [54] developed ovarian cancer PDXs in female SCID/beige mice, which were divided into two cohorts: high PAPP-A $(\mathrm{n}=5)$ group and low PAPP-A $(n=2)$ group, and then injected these models with saline, carboplatin/paclitaxel + anti- PAPP-A monoclonal antibody inhibitor (mAb-PA), or carboplatin/paclitaxel + IgG2a (control antibody) in randomization. They then examined these models with ultrasound after 28 days of treatment and found that carboplatin/paclitaxel combined with mAb-PA induced tumor degeneration below baseline in one high PAPP-A PDX model and inhibited tumor growth in another three models compared with carboplatin/ paclitaxel + IgG2a, while no low PAPP-A PDX models regressed tumor below baseline, suggesting that mAb-PA sensitized ovarian cancer to carboplatin/ paclitaxel chemotherapy. In line with this, Garrett et al. [55] established HGS-OC PDX models by transplanting tumor tissues into 6-week old female NOD/SCID mice, which then treated with carboplatin/paclitaxel for 21 days, and found that LBH589, a novel histone deacetylase inhibitor panobinostat, decreased the growth of ovarian cancer in PDXs and promoted the effect of carboplatin/ paclitaxel therapy in one of three PDX models. Another group developed 14 clinically annotated and molecularly characterized luciferized ascites-derived ovarian cancer PDX models, and demonstrated the consistency of response to carboplatin and paclitaxel evaluation across different assay platforms, including bioluminescent imaging (BLI) and plasma CA125 levels or LINE-1 biomarkers, suggesting that BLI can be as a platform for proof-of-concept efficacy and biomarker studies and for validation of novel therapeutic strategies in ovarian cancer [56].

Another utility of PDX models for standard chemotherapy is to explore biomarkers or pathways related to chemo-resistance that may help understand the chemoresistance mechanisms. One group established 42 ovarian cancer PDX models with different sensitivity to cisplatin, revealing that cyclin dependent kinase 12 (CDK12) mRNA expression was negatively related to cisplatin sensitivity and positively associated with tumor recurrence rate in high-grade serous/endometrioid ovarian cancer PDX samples in vivo, suggesting that the CDK12 may be an important gene in ovarian cancer cell resistance to cisplatin [57]. Moreover, Wnt/ $\beta$-catenin signaling pathway was demonstrated to be associated with platinum resistance of HGS-OC PDX models, with retention of stem-like properties, implying that Wnt/ $\beta$-catenin inhibitor iCG-001 induced cisplatin chemosensitivity of ovarian cancer cells [58]. In addition, $\mathrm{Li}$ et al. [59] established 3 chemoresistance and 4 chemosensitivity HGS-OC PDX tumor models by implanting cancer tissues subcutaneously into mice which were then treated with paclitaxel and carboplatin. In these models, they identified 146 upregulated genes and 54 down-regulated genes in chemoresistance group, including genes SAP25, HLA-DPA1, AKT3, and PIK3R5 by RNA sequencing analysis, and also found 39 mutation sites only shown in chemoresistance group, including important gene mutation of TMEM205 and POLR2A by whole exome sequencing analysis, suggesting these differently expressed genes and mutations could be provided to predict chemotherapy response.

\section{Targeted therapy of PDX models in gynecologic cancers}

Accumulating evidence has demonstrated that PDXs play a vital role in the trial of targeted therapeutic drugs, which helps provide individual treatment for gynecologic cancer patients.

\section{PARP inhibitors}

Up to date, although polyadenosine diphosphate ribose polymerase (PARP) inhibitors (PARPi) have been applied for clinical therapy for many years, the therapeutic resistance to them especially in ovarian cancer treatment is still a clinical problem. Herein, more and more researchers turn the PARPi study to the PDX models. Nowadays, FDA-approved PARP inhibitors for treating HGS-OC include olaparib, rucaparib and niraparib [60]. Almost half of the EOCs exhibit defective DNA repair via homologous 
recombination containing BRCA1/2 (BRCA1 and BRCA2) mutations and formation of Rad51 foci after DNA damage. Homologous recombination deficiency is an important target for PARPi to treat ovarian cancer [61]. To date, an increasing number of researchers have utilized PDX models to study the biomarkers related to the resistance to PARPi in ovarian cancers. For example, it was found that methylation of all BRCA1 copies was correlated with the response to the PARPi rucaparib in 12 BRCA1methylated HGS-OC models [62]. Shah et al. [63] examined 3 ovarian cancer PDX models by implanting fresh omental ovarian tumor nodules into SCID mice and showed that the PDXs' response to PARPi was related to the ex vivo ionizing radiation (IR) assay. Notably, an increased expression of euchromatic histone-lysine-N-methyltransferases 1 and 2 (EHMT1/2, akaGLP/G9A) has been validated in PARPi-resistant HGS-OC PDX models, implying its association with the resistance to PARPi of HGS-OC [64].

Parmar et al. [65] utilized 14 characterized luciferized HGS-OC PDX models and revealed that 13 models were resistant to olaparib monotherapy, among which 4 models presenting BRCA1 mutation, and olaparib in combination with a checkpoint kinase 1 (CHK1) inhibitor prexasertib contributed to tumor inhibition in olaparib-resistant models. Consistently, it was identified that combined use of PARPi and ATR/CHK1 inhibitor exhibited a more effective antitumor activity than PARPi monotherapy in the established recurrent BRCA-mutant (BRCAMUT) HGS-OC PDX models [66]. Whilst, olaparib and chloroquine (CQ) were shown to induce synergistic antitumor activity and promoted drug resistance via autophagy in ovarian cancer PDX models [67]. In contrast, AlHilli et al. [68] developed five homologous recombination deficiency HGS-OC PDX models intraperitoneally in 35 female SCID mice to evaluate the antitumor role of niraparib, and found that niraparib monotherapy in one of two PDX models with deficient BRCA2 and in one PDX with Rad51C promoter methylation induced cancer regression, although these models were failed to promote response to carboplatin/paclitaxel chemotherapy.

\section{VEGF inhibitor}

Bevacizumab is a monoclonal antibody against vascular endothelial growth factor A (VEGFA), and has been widely used in cancer second line therapy as an anti-angiogenic drug. A research using three cisplatin-relapsing ovarian cancer PDX models based on the presence of activation of the RAS/RAF/MEK/ ERK axis and PI3K pathway, p53 mutation and lack of phosphatase and tensin homolog (PTEN) expression, confirmed that triple combination of bevacizumab, MEK162 (a MEK inhibitor) and paclitaxel displayed a more effective antitumor activity than any double drug combination for relapsing ovarian tumors in second line treatment [69]. Meanwhile, another study used 11 EOC PDXs by transplanting tumor tissues orthotopically in the peritoneal cavity of nude mice to evaluate the activity of cediranib (a pan-VEGFR RTK inhibitor) alone or combined with chemotherapy, showing that different EOC PDX models displayed dissimilar response to cediranib, and that combination of cediranib and cisplatin increased the model mice survival and inhibited ascites and metastases while cediranib alone just exerted the decreasing effect on ascites but not on tumor dissemination in advanced EOC PDX [70].

\section{$\mathrm{PI3K} / \mathrm{Akt} / \mathrm{mTOR}$ inhibitor}

Several inhibitors of PI3K/Akt/mTOR pathway have been clinically applied to some malignancies including ovarian cancer, and c-Met receptor has been found to promote PI3K/Akt/mTOR pathway and to play an important role in drug resistance [71]. Activation of $\mathrm{PI} 3 \mathrm{~K} / \mathrm{Akt} / \mathrm{mTOR}$ pathway has been identified to be involved in chemotherapy resistance or anti-EGFR/HER2 therapies, and PTEN inhibition activates the PI3K/Akt/mTOR pathway. In a lowgrade serous ovarian cancer (LGSOC) PDX model with lack of PTEN expression, two PI3K/Akt/mTOR inhibitors (PF-04691502 and PF-05212384) added to cisplatin or paclitaxel promoted the activity of chemotherapy alone in LGSOC models, suggesting that PI3K-mTOR inhibitors contributed to the chemotherapy sensitivity to cisplatin or paclitaxel [72]. An in vivo study in one ovarian cancer PDX model revealed that the combination of crizotinib (a c-Met inhibitor) and gedatolisib (a dual PI3K-mTOR inhibitor) exerted a more superior antitumor activity than single agent, while crizotinib alone presented no antitumor activity, and gedatolisib alone only displayed some marginal activity, suggesting that crizotinib promoted the activity of gedatolisib in treating ovarian cancer [73]. Another research using a large cohort of human uterine sarcoma samples (288) and identified the most promising target phospho-S6 ribosomal protein ( $\mathrm{p}-\mathrm{S} 6$ ) among 5 common druggable targets. In order to investigate this target, they developed 5 leiomyosarcoma PDX models and uncovered that PI3K/mTOR inhibitors (BEZ235, also known as dactolisib) therapy inhibited cancer growth of 4/5 leiomyosarcoma PDX models, and the 4 responding models presented basal p-S6 expression but nonresponding model showed negative score, suggesting that dual $\mathrm{PI} 3 \mathrm{~K} / \mathrm{mTOR}$ inhibition is a promising therapeutic strategy in uterine 
leiomyosarcoma, and p-S6 expression can be used for predicting its response [74].

\section{RTK inhibitor}

Receptor tyrosine kinases (RTKs) are a subclass of tyrosine kinases that catalyse the transfer of a phosphate from ATP to a hydroxyl group of a tyrosine residue. Nowadays, 58 RTKs have been identified in human beings, and classified to 20 subfamilies, such as receptors of ERBB, insulin, platelet derived growth factor (PDGF) and vascular endothelial growth factor (VEGF), playing an important role in cancer progression [75,76]. Therefore, RTKs inhibitors are frequently used to treat tumors. Ponatinib is a small molecule multi-tyrosine kinase inhibitor clinically approved for anticancer therapy. One research group established a rare malignant ovarian cancer small cell carcinoma of the ovary hypercalcemic type (SCCOHT) PDX model and demonstrated that ponatinib delayed tumor doubling time and decreased final tumor volume in this PDX model by $58.6 \%$ and $42.5 \%$, respectively [77]. In addition, erlotinib is also a RTK inhibitor, which inhibited the activation of epidermal growth factor receptor (EGFR), and has been administrated to treat many cancers. It exerted its antitumor function by inhibiting angiogenesis and consequently impairing intratumoral microcirculation [78]. Another research group established an EGFR-overexpressing clear cell ovarian carcinoma PDX, and indicated that erlotinib markedly reduced tumor weight in this model [38].

\section{HER2 inhibitor}

HER2 belongs to the epidermal growth factor (ErbB, HER) family and is encoded by proto-oncogene ERBB2 on chromosome 17 [79]. Activated HER2 can promote cancer cell growth and survival and induce reprogramming of tumor metabolism. The HER2 receptor is overexpressed in various cancers, being an attractive target in cancer therapy. Trastuzumab and lapatinib, as US Federal Drug Administrationapproved HER2 inhibitors, are commonly used to administrate HER2-overexpressing breast cancers. In 14 established HER2-amplified cervical cancer PDX models, combined treatment of trastuzumab and lapatinib reduced $50 \%$ of PDX tumor weight compared with the untreated control, identifying that trastuzumab and lapatinib suppressed cancer growth in the HER2-overexpressed PDXs [19]. A recent study transplanted fresh specimens from naïve-treatment ovarian cancer females intraperitoneally into female SCID beige mice to establish 3 ovarian cancer PDX models and then grouped them into untreated, pertuzumab treated, and carboplatin/paclitaxel treated groups, discovering that pertuzumab inhibited tumor growth but did not induce tumor regression, and carboplatin/paclitaxel chemotherapy decreased about $25 \%$ of tumor volume, compared with untreated group increasing tumor volume with 4-4.5 fold over the 4 weeks. Meanwhile, the coadministration of HER2-targeted therapy and carboplatin/paclitaxel chemotherapy contributed to a significant tumor regression after 6 weeks in comparison with single chemotherapy, suggesting that HER2-targeted therapy sensitized ovarian cancer response to chemotherapy of carboplatin and paclitaxel [27].

For observing the anti-metastatic function of HER2-targeted therapy, it is important to develop orthotopic PDX models since metastatic and primary tumors in an orthotopic model may exert differential chemosensitivity $[80,81]$. For instance, a benzamide histone deactylase inhibitor entinostat displayed no tumor inhibition in HER-2 expressing cervical carcinoma PDX model in a subcutaneous nude mouse, and also did not suppress original tumor growth in the orthotopic PDX model. But, entinostat alone notably inhibited the metastatic tumor burden in comparison with the control [80].

\section{Other target therapy}

Gemcitabine is an analog of deoxycytidine, and has been widely used for anticancer treatment. Trabectedin is a cytotoxic agent derived from Caribbean sea squirt with the antitumor activity in treating several cancers including ovarian cancer [82]. Pegylated liposomal doxorubicin (PLD) is a formulation of doxorubicin encapsulated in liposomes as an anthracycline topoisomerase inhibitor and has been commonly used for the treatment of many solid malignancies, including recurrent or progressive gynecologic cancers [83]. Erriquezet al. [84] implanted pairs of HGS-OC samples from the same patients before and after platinum-based neo-adjuvant chemotherapy into immunocompromised mice, and then treated these xenograft mice equally with carboplatin, gemcitabine, PLD and trabectedin, showing that naïve HSG-OC PDX models displayed response to carboplatin, trabectedin, gemcitabine and PLD, while carboplatin treated PDXs propagated from a tumor mass of the same patient, lost response to trabectedin, gemcitabine and PLD. It suggested that ovarian cancer having been treated with chemotherapeuticc drug may presents chemo-resistance to second line chemotherapy.

\section{Immunotherapy response of PDX models in gynecologic cancers}

With the emerging of tumor immunotherapies and vaccines for tumor therapy, PDX models potently 
evaluating the preclinical activity of these immunotherapies are more and more important. However, during the development of these PDX models for studying immunotherapies, the unintended formation of human lymphoma is being a potential problem. Butler et al. [85] established ovarian cancer PDX models by injecting species from 568 ovarian cancer patients intraperitoneally into SCID mice and indicated that rituximab, an anti-CD20 antibody, decreased CD45-positive cells incidence in subsequent PDX lines from $86.3 \%$ (no rituximab) to $5.6 \%$ (rituximab), and reduced lymphoma rate from $11.1 \%$ to $1.88 \%$.

Chimeric antigen receptor (CAR) - $\mathrm{T}$ cells treatment may be ineffective for solid tumors, since vascular barriers hamper CAR-T cells from reaching the tumor site. Deng et al. [86] used subcutaneous ovarian cancer mouse xenograft models and ovarian cancer PDX models, and reported that combretastatin A-4 phosphate (CA4P), a vascular disrupting agent (VDA), significantly enhanced the therapeutic efficiency of the CAR-T cells, providing a new potential strategy for CAR-T cells treatment in ovarian cancer.

Using RNASeq analyses, another study identified the upregulation of antigen presenting pathways in both ovarian cancer PDXs and original tumors, hinting a strong functional conservation between them. Among the tested 30 neoantigens, they discovered that a core of six neoantigens defining a potent autologous $\mathrm{T}$ cell activation inhibited cancer growth [87]. Similarly, recent study used orthotopically transplanted HGS-OC tissues and matched autologous-expanded tumor infiltrating lymphocytes (TILs) into NSG mice to create humanized TIL/PDX models for evaluating the anti-tumor effect of immunomodulating therapies against autologoustumors. In this study, the mice were accepted with TIL infusion alone, TIL infusion + anti programmed cell death protein 1 (PD-1), or vehicle, and mice treated with TILs and anti-PD-1 decreased tumor volume and increased overall survival [46]. Another group utilized ovarian cancer PDX models and found a promising therapeutic target Ephrin-A4 (EFNA4) with the identification of E-cadherin (CD324) as a surface antigen able to enrich TIC, and revealed that an antibody-drug conjugate (ADC) containing antiEFNA4 monoclonal antibody conjugated to the DNAdamaging agent calicheamicin inhibited tumor growth [88]. The results of these studies suggest that PDX models of gynecologic cancers especially ovarian cancers play important roles in evaluating human response to immunotherapy treatment and designing optimal clinical trials.

\section{Other therapies of PDX models in gynecologic cancers}

CX-5461 is an RNA polymerase I (Pol I) inhibitor, which exerts its action by inhibiting ribosomal DNA transcription. For studying its antitumor activity in ovarian cancer, one group established 5 ovarian cancer PDXs from 5 advanced papillary serous ovarian cancer patients and uncovered that these models displayed differential response to CX-5461 treatment, with complete response in 1 model, 55\% reduction in cancer volume in 1 model, stable disease in 1 model and tumor growing in 2 models after 45 days [89], suggesting that more PDX models are need to carry out, and potential biomarkers are required to find in the study of ovarian cancer response to CX-5461. CUB-domain containing protein 1 (CDCP1) is a cell-surface protein and has been identified to be overexpressed in multiple tumors including clear cell ovarian cancer. It was noticed that in 3 established HGS-OC PDXs, the antibody to CDCP1 dramatically inhibited cancer growth in these ovarian cancer PDX models [90]. Furthermore, sphingosine kinase 1 (SK1) inhibitor, FTY720, has been indicated to dramatically attenuate tumor weight in ovarian cancer cell lines (A2780 and SKOV3ip1) xenograft models and a clear cell ovarian carcinoma (CCC) PDX model [91], as well as in cervical cancer PDX models [92], supporting FTY720 as a potential therapeutic agent for gynecologic cancers. Furthermore, two EOC PDX models were used to confirm the activity of itraconazole in ovarian cancers and revealed that combined treatment of itraconazole and paclitaxel markedly attenuated cancer weight, decreased microvessel density of PDX tumor as well as suppressed hedgehog and mTOR pathways in comparison with the control, paclitaxel-alone, or itraconazole-alone groups, suggesting that itraconazole suppressed endothelial cells rather than cancer cells by targeting several signaling pathways including angiogenesis, hedgehog and mTOR pathways [93].

\section{PDX models for discovering new therapeutic drugs in gynecologic cancers}

Another utility of PDX models in gynecologic cancers is to discover novel therapeutic drugs, with greater predictive value for incipient drug testing. In 2015, considering the activity of Mullerian inhibiting substance (MIS) in suppressing the growth of stemlike ovarian cancer cells, one research group devised peptide modifications to human MIS (LRMIS) and delivered it with adeno-associated virus (AAV) to validate its anti-tumor function in chemoresistant serous ovarian adenocarcinoma PDX models from ascites, showing that AAV9-LRMIS monotherapy 
notably reduced cancer growth without signs of toxicity in 3/5 these PDXs, hinting AAV9-LRMIS as an effective agent for chemoresistant serous ovarian cancer patients [94]. Cell division cycle 25B (CDC25B) has been identified to be correlated with poor prognosis of ovarian cancer. BAY-876, as a newgeneration inhibitor of glucose transporter 1 (GLUT1), is overexpressed in ovarian cancer but has not been assessed in preclinical animal models. With the use of ovarian cancer PDXs, CDC25B inhibitor WG-391D [95], and BAY-876 [96] have been proved to regress ovarian cancer growth in PDXs.

\section{PDX models for studying mechanism of gynecologic cancer}

PDX models also exhibit a critical role in researching cancer mechanism and biomarkers related to cancer development. SET and MYND domain-containing protein 3 (SMYD3), a histone methyltransferase, is a promising epigenetic therapeutic target and has been found to be upregulated in a variety of human cancers. Using ovarian cancer PDX models, SMYD3 was identified to induce ovarian cancer growth [97], and to promote metastasis and reduce ascites volume in ovarian cancer PDX models [98], suggesting that SMYD3 may be related to the development of ovarian cancer. Additionally, an in vivo research using cell-line xenograft and EOC PDX models revealed that cyclindependent kinase 7 (CDK7) increased ovarian cancer weight via regulation of cell proliferation and apoptosis [99]. Of note, Erriquezet al. [100] utilized ovarian cancer PDX models and found an overexpression of topoisomerase II alpha (TOP2A) in cells from xenograft after the treatment of pegylated liposomal doxorubicin (PLD), suggesting that TOP2A may be involved in the pathogenesis of ovarian cancer response to PLD.

\section{Conclusions}

Current evidence has demonstrated that gynecologic cancer PDX model is a useful tool for predicting response to chemotherapy, targeted therapy, immunotherapy, as well as discovering new drugs, for better designing optimal clinical trials, because of its high concordance with original tumor in phenotypic and proteomic characteristics, gene expression, and drug response. However, when it is hoped to provide personalized and precise therapy to each individual cancer patient using PDX models, some limitations and challenges must be mentioned and overcome, which include variable engraftment rates, enormous time and resources taken for PDX generation, high cost, loss of immune systems, and uncertainty of the effect of tumor microenvironment on drug efficacy. Furthermore, except for ovarian cancer PDXs, uterine cancer and other gynecologic cancer PDX models are so few that they cannot benefit these patients with personalized treatment regime. Herein, international collaborative networks should work together to overcome these drawbacks for making the real personalized treatment to come true.

\section{Abbreviations}

EOC: epithelial ovarian cancer; HGS-OC: high-grade serous ovarian carcinoma; HG-OC: high grade ovarian cancer; ER: estrogen receptor; PR: progesterone receptor; TSP-1: thrombospondin-1; ROR1: receptor tyrosine kinase-like orphan receptor; SCID: severe combined immuno-deficient; NOD: non-obese diabetic; TCGA: The Cancer Genome Atlas.

\section{Acknowledgements}

This work was sponsored by Science and Technology Planning Project of Wenzhou city (No.ZS2017006).

\section{Competing Interests}

The authors have declared that no competing interest exists.

\section{References}

1. Siegel RL, Miller KD, Jemal A. Cancer statistics, 2020. CA Cancer J Clin. 2020;70(1):7-30.

2. Sa JK, Hwang JR, Cho YJ, et al. Pharmacogenomic analysis of patient-derived tumor cells in gynecologic cancers. Genome Biol. 2019;20(1):253.

3. Cohen PA, Jhingran A, Oaknin A, Denny L. Cervical cancer. Lancet. 2019;393(10167):169-182.

4. Webb PM, Jordan SJ. Epidemiology of epithelial ovarian cancer. Best Pract Res Clin Obstet Gynaecol. 2017;41:3-14.

5. Mestas J, Hughes CC. Of mice and not men: differences between mouse and human immunology. J Immunol. 2004;172(5):2731-2738.

6. Wege AK. Humanized Mouse Models for the Preclinical Assessment of Cancer Immunotherapy. Bio Drugs. 2018;32(3):245-266.

7. Kojima Y, Hayakawa F, Morishita T, et al. YM155 induces apoptosis through proteasome-dependent degradation of MCL-1 in primary effusion lymphoma. Pharmacol Res. 2017;120:242-251.

8. Wang $\mathrm{Z}, \mathrm{Fu} \mathrm{S}$, Zhao J, et al. Transbronchoscopic patient biopsy-derived xenografts as a preclinical model to explore chemorefractory-associated pathways and biomarkers for small-cell lung cancer. Cancer Lett. 2019;440-441:180-188.

9. Kuwata T, Yanagihara K, Iino Y, et al. Establishment of Novel Gastric Cancer Patient-Derived Xenografts and Cell Lines: Pathological Comparison between Primary Tumor, Patient-Derived, and Cell-Line Derived Xenografts. Cells. 2019;8(6):585.

10. Stewart EL, Mascaux C, Pham NA, et al. Clinical Utility of Patient-Derived Xenografts to Determine Biomarkers of Prognosis and Map Resistance Pathways in EGFR-Mutant Lung Adenocarcinoma. J ClinOncol. 2015;33(22):2472-2480

11. Su D, Zhang D, Jin J, et al. Identification of predictors of drug sensitivity using patient-derived models of esophageal squamous cell carcinoma. Nat Commun. 2019;10(1):5076

12. Bello E, Brich S, Craparotta I, et al. Establishment and characterisation of a new patient-derived model of myxoid liposarcoma with acquired resistance to trabectedin. Br J Cancer. 2019:121(6):464-473.

13. Wang J, Xing B, Liu W, et al. Molecularly annotation of mouse avatar models derived from patients with colorectal cancer liver metastasis. Theranostics. 2019;9(12):3485-3500.

14. Yang X, Meng G. Establishment of a non-small-cell lung cancer-liver metastasis patient-derived tumor xenograft model for the evaluation of patient-tailored chemotherapy. Biosci Rep. 2019;39(7): BSR20182082.

15. Vaeteewoottacharn K, Pairojkul C, Kariya R, et al. Establishment of Highly Transplantable Cholangiocarcinoma Cell Lines from a Patient-Derived Xenograft Mouse Model. Cells. 2019;8(5):496. 
16. Wu J, Zheng Y, Tian Q, Yao M, Yi X. Establishment of patient-derived xenograft model in ovarian cancer and its influence factors analysis. J Obstet Gynaecol Res. 2019;45(10):2062-2073.

17. Yang W, Fan WS, Ye MX, et al. Establishment of the PDTX model of gynecological tumors. Am J Transl Res. 2019;11(6):3779-3789.

18. Larmour LI, Cousins FL, Teague JA, Deane JA, Jobling TW, Gargett CE. A patient derived xenograft model of cervical cancer and cervical dysplasia. PLoS One. 2018;13(10):e0206539.

19. Oh DY, Kim S, Choi YL, et al. HER2 as a novel therapeutic target for cervical cancer. Oncotarget. 2015;6(34):36219-36230.

20. Scott CL, Mackay HJ, Haluska P, Jr. Patient-derived xenograft models in gynecologic malignancies. Am Soc Clin Oncol Educ Book. 2014:e258-266.

21. Lai Y, Wei X, Lin S, et al. Current status and perspectives of patient-derived xenograft models in cancer research. J Hematol Oncol. 2017;10(1):106.

22. Okada S, Vaeteewoottacharn K, Kariya R. Application of Highly Immunocompromised Mice for the Establishment of Patient-Derived Xenograft (PDX) Models. Cells. 2019;8(8):889.

23. Wang Y, Wang JX, Xue H, et al. Subrenal capsule grafting technology in human cancer modeling and translational cancer research. Differentiation. 2016;91(4-5):15-19.

24. Hoffman RM. Patient-derived orthotopic xenografts: better mimic of metastasis than subcutaneous xenografts. Nat Rev Cancer. 2015;15(8):451-452.

25. Morton CL, Houghton PJ. Establishment of human tumor xenografts in immunodeficient mice. Nat Protoc. 2007;2(2):247-250.

26. Bratcher NA, Frost DJ, Hickson J, et al. Effects of Buprenorphine in a Preclinical Orthotopic Tumor Model of Ovarian Carcinoma in Female CB17 SCID Mice. J Am Assoc Lab Anim Sci. 2019;58(5):583-588.

27. Harris FR, Zhang $\mathrm{P}$, Yang $\mathrm{L}$, et al. Targeting HER2 in patient-derived xenograft ovarian cancer models sensitizes tumors to chemotherapy. Mol Oncol. 2019;13(2):132-152.

28. Zhu M, Jia N, Nie Y, et al. Establishment of Patient-Derived Tumor Xenograft Models of High-Risk Endometrial Cancer. Int J Gynecol Cancer. 2018;28(9):1812-1820

29. Hauge A, Wegner CS, Gaustad JV, Simonsen TG, Andersen LMK, Rofstad EK. Diffusion-weighted MRI-derived ADC values reflect collagen I content in PDX models of uterine cervical cancer. Oncotarget. 2017;8(62):105682-105691.

30. Hauge A, Wegner CS, Gaustad JV, Simonsen TG, Andersen LMK, Rofstad EK. DCE-MRI of patient-derived xenograft models of uterine cervix carcinoma: associations with parameters of the tumor microenvironment. J Transl Med. 2017;15(1):225

31. Haldorsen IS, Popa M, Fonnes T, et al. Multimodal Imaging of Orthotopic Mouse Model of Endometrial Carcinoma. PLoS One. 2015;10(8):e0135220.

32. Depreeuw J, Hermans E, Schrauwen S, et al. Characterization of patient-derived tumor xenograft models of endometrial cancer for preclinical evaluation of targeted therapies. Gynecol Oncol. 2015:139(1):118-126.

33. Pauli C, Hopkins BD, Prandi D, et al. Personalized In vitro and In vivo Cancer Models to Guide Precision Medicine. Cancer Discov. 2017;7(5):462-477.

34. Colombo PE, du Manoir S, Orsett B, et al. Ovarian carcinoma patient derived xenografts reproduce their tumor of origin and preserve an oligoclonal structure. Oncotarget. 2015;6(29):28327-28340.

35. Weroha SJ, Becker MA, Enderica-Gonzalez S, et al. Tumor grafts as in vivo surrogates for women with ovarian cancer. Clin Cancer Res. 2014;20(5):1288-1297.

36. Boone JD, Dobbin ZC, Straughn JM Jr, Buchsbaum DJ, et al. Ovarian and cervical cancer patient derived xenografts: The past, present, and future. Gynecol Oncol. 2015;138(2):486-491.

37. Cuppens T, Depreeuw J, Annibali D, et al. Establishment and characterization of uterine sarcoma and carcinosarcoma patient-derived xenograft models. Gynecol Oncol. 2017;146(3):538-545.

38. Heo EJ, Cho YJ, Cho WC, et al. Patient-Derived Xenograft Models of Epithelial Ovarian Cancer for Preclinical Studies. Cancer Res Treat. 2017;49(4):915-926.

39. Colon-Otero G, Weroha SJ, Foster NR, et al. Phase 2 trial of everolimus and letrozole in relapsed estrogen receptor-positive high-grade ovarian cancers. Gynecol Oncol. 2017;146(1):64-68.

40. Topp MD, Hartley L, Cook M, et al. Molecular correlates of platinum response in human high-grade serous ovarian cancer patient-derived xenografts. Mol Oncol. 2014;8(3):656-668

41. Taghian A, Budach W, Zietman A, et al. Quantitative comparison between the transplantability of human and murine tumors into the subcutaneous tissue of $\mathrm{NCr} /$ Sed-nu/nu nude and severe combined immunodeficient mice. Cancer Res. 1993;53(20):5012-5017

42. Collins AT, Lang SH. A systematic review of the validity of patient derived xenograft (PDX) models: the implications for translational research and personalised medicine. Peer J. 2018;6:e5981.

43. Alkema NG, Tomar T, Duiker EW, et al. Biobanking of patient and patient-derived xenograft ovarian tumour tissue: efficient preservation with low and high fetal calf serum based methods. Sci Rep. 2015;5:14495.

44. Chen Y, Zhang R, Wang L, et al. Tumor characteristics associated with engraftment of patient-derived non-small cell lung cancer xenografts in immunocompromised mice. Cancer. 2019;125(21):3738-3748.

45. Zhang S, Cui B, Lai H, et al. Ovarian cancer stem cells express ROR1, which can be targeted for anti-cancer-stem-cell therapy. Proc Natl Acad Sci U S A. 2014;111(48):17266-17271.
46. Gitto SB, Kim H, Rafail $S$, et al. An autologous humanized patient-derived-xenograft platform to evaluate immunotherapy in ovarian cancer. Gynecol Oncol. 2020; 156(1):222-232.

47. Pinessi D, Ostano P, Borsotti $\mathrm{P}$, et al. Expression of thrombospondin- 1 by tumor cells in patient-derived ovarian carcinoma xenografts. Connect Tissue Res. 2015;56(5):355-363.

48. Liu Y, Chanana P, Davila JI, et al. Gene expression differences between matched pairs of ovarian cancer patient tumors and patient-derived xenografts. Sci Rep. 2019;9(1):6314.

49. Cybulska P, Stewart JM, Sayad A, et al. A Genomically Characterized Collection of High-Grade Serous Ovarian Cancer Xenografts for Preclinical Testing. Am J Pathol. 2018;188(5):1120-1131.

50. Dobbin ZC, Katre AA, Steg AD, et al. Using heterogeneity of the patient-derived xenograft model to identify the chemoresistant population in ovarian cancer. Oncotarget. 2014;5(18):8750-8764.

51. Glaser G, Weroha SJ, Becker MA, et al. Conventional chemotherapy and oncogenic pathway targeting in ovarian carcinosarcoma using a patient-derived tumor graft. PLoS One. 2015;10(5):e0126867.

52. Byeon Y, Lee JW, Choi WS, et al. CD44-Targeting PLGA Nanoparticles Incorporating Paclitaxel and FAK siRNA Overcome Chemoresistance in Epithelial Ovarian Cancer. Cancer Res. 2018;78(21):6247-6256.

53. Chen Y, Camacho SC, Silvers TR, et al. Inhibition of the Nuclear Export Receptor XPO1 as a Therapeutic Target for Platinum-Resistant Ovarian Cancer. Clin Cancer Res. 2017;23(6):1552-1563.

54. Torres D, Hou X, Bale L, et al. Overcoming platinum resistance in ovarian cancer by targeting pregnancy-associated plasma protein-A. PLoS One. 2019;14(11):e0224564.

55. Garrett LA, Growdon WB, Rueda BR, Foster R. Influence of a novel histone deacetylase inhibitor panobinostat (LBH589) on the growth of ovarian cancer. J Ovarian Res. 2016;9(1):58.

56. Liu JF, Palakurthi S, Zeng O, et al. Establishment of Patient-Derived Tumor Xenograft Models of Epithelial Ovarian Cancer for Preclinical Evaluation of Novel Therapeutics. Clin Cancer Res. 2017;23(5):1263-1273.

57. Guffanti F, Fratelli M, Ganzinelli M, et al. Platinum sensitivity and DNA repair in a recently established panel of patient-derived ovarian carcinoma xenografts. Oncotarget. 2018;9(37):24707-24717.

58. Nagaraj $A B$, Joseph $P$, Kovalenko $O$, et al. Critical role of Wnt/beta-catenin signaling in driving epithelial ovarian cancer platinum resistance. Oncotarget. 2015;6(27):23720-23734

59. Li LY, Kim HJ, Park SA, et al. Genetic Profiles Associated with Chemoresistance in Patient-Derived Xenograft Models of Ovarian Cancer. Cancer Res Treat. 2019;51(3):1117-1127.

60. Matulonis UA. Management of newly diagnosed or recurrent ovarian cancer. Clin Adv Hematol Oncol. 2018;16(6):426-437.

61. Konstantinopoulos PA, Ceccaldi R, Shapiro GI, D'Andrea AD. Homologous Recombination Deficiency: Exploiting the Fundamental Vulnerability of Ovarian Cancer. Cancer Discov. 2015;5(11):1137-1154.

62. Kondrashova O, Topp M, Nesic K, et al. Methylation of all BRCA1 copies predicts response to the PARP inhibitor rucaparib in ovarian carcinoma. Nat Commun. 2018;9(1):3970

63. Shah MM, Dobbin ZC, Nowsheen S, et al. An ex vivo assay of XRT-induced Rad51 foci formation predicts response to PARP-inhibition in ovarian cancer. Gynecol Oncol. 2014;134(2):331-337.

64. Watson ZL, Yamamoto TM, McMellen A, et al. Histone methyltransferases EHMT1 and EHMT2 (GLP/G9A) maintain PARP inhibitor resistance in high-grade serous ovarian carcinoma. Clin Epigenetics. 2019;11(1):165.

65. Parmar K, Kochupurakkal BS, Lazaro JB, et al. The CHK1 Inhibitor Prexasertib Exhibits Monotherapy Activity in High-Grade Serous Ovarian Cancer Models and Sensitizes to PARP Inhibition. Clin Cancer Res. 2019;25(20):6127-6140.

66. Kim H, George E, Ragland R, et al. Targeting the ATR/CHK1 Axis with PARP Inhibition Results in Tumor Regression in BRCA-Mutant Ovarian Cancer Models. Clin Cancer Res. 2017:23(12):3097-3108.

67. Santiago-O'Farrill JM, Weroha SJ, Hou X, et al. Poly (adenosine diphosphate ribose) polymerase inhibitors induce autophagy-mediated drug resistance in ovarian cancer cells, xenografts, and patient-derived xenograft models. Cancer. 2020; 126(4):894-907.

68. AlHilli MM, Becker MA, Weroha SJ, et al. In vivo anti-tumor activity of the PARP inhibitor niraparib in homologous recombination deficient and proficient ovarian carcinoma. Gynecol Oncol. 2016;143(2):379-388.

69. Ricci F, Guffanti F, Damia G, Broggini M. Combination of paclitaxel, bevacizumab and MEK162 in second line treatment in platinum-relapsing patient derived ovarian cancer xenografts. Mol Cancer. 2017;16(1):97.

70. Decio A, Cesca M, Bizzaro F, et al. Cediranib combined with chemotherapy reduces tumor dissemination and prolongs the survival of mice bearing patient-derived ovarian cancer xenografts with different responsiveness to cisplatin. Clin Exp Metastasis. 2015;32(7):647-658.

71. Maroun CR, Rowlands T. The Met receptor tyrosine kinase: a key player in oncogenesis and drug resistance. PharmacolTher. 2014;142(3):316-338.

72. Brana I, Pham NA, Kim L, et al. Novel combinations of PI3K-mTOR inhibitors with dacomitinib or chemotherapy in PTEN-deficient patient-derived tumor xenografts. Oncotarget. 2017;8(49):84659-84670.

73. Iezzi A, Caiola E, Broggini M. Activity of Pan-Class I Isoform PI3K/mTOR Inhibitor PF-05212384 in Combination with Crizotinib in Ovarian Cancer Xenografts and PDX. Transl Oncol. 2016;9(5):458-465. 
74. Cuppens T, Annibali D, Coosemans A, et al. Potential Targets' Analysis Reveals Dual PI3K/mTOR Pathway Inhibition as a Promising Therapeutic Strategy for Uterine Leiomyosarcomas-an ENITEC Group Initiative. Clin Cancer Res. 2017;23(5):1274-1285.

75. Lemmon MA, Schlessinger J. Cell signaling by receptor tyrosine kinases. Cell. 2010;141(7):1117-1134.

76. Butti R, Das S, Gunasekaran VP, et al. Receptor tyrosine kinases (RTKs) in breast cancer: signaling, therapeutic implications and challenges. Mol Cancer. 2018;17(1):34.

77. Lang JD, Hendricks WPD, Orlando KA, et al. Ponatinib Shows Potent Antitumor Activity in Small Cell Carcinoma of the Ovary Hypercalcemic Type (SCCOHT) through Multi kinase Inhibition. Clin Cancer Res. 2018;24(8):1932-1943.

78. Selcukbiricik F, Ozdogan E, Dagel T, et al. Elevation in serum uric acid levels predicts favourable response to erlotinib treatment in patients with metastatic non-small-cell lung cancer. J Clin Pharm Ther. 2020; 45(2):303-308.

79. Akiyama T, Sudo C, Ogawara H, et al. The product of the human c-erbB-2 gene: a 185-kilodalton glycoprotein with tyrosine kinase activity. Science. 1986;232(4758):1644-1646

80. Hiroshima $\mathrm{Y}$, Maawy A, Zhang $\mathrm{Y}$, et al. Patient-derived mouse models of cancer need to be orthotopic in order to evaluate targeted anti-metastatic therapy. Oncotarget. 2016;7(44):71696-71702.

81. Hiroshima Y, Zhang Y, Zhang N, et al. Establishment of a patient-derived orthotopic Xenograft (PDOX) model of HER-2-positive cervical cancer expressing the clinical metastatic pattern. PLoS One. 2015;10(2):e0117417.

82. Souid S, Aissaoui D, Srairi-Abid N, Essafi-Benkhadir K. Trabectedin (Yondelis(R)) as a Therapeutic Option in Gynecological Cancers: A Focus on its Mechanisms of Action, Clinical Activity, and Genomic Predictors of Drug Response. Curr Drug Targets 2020; [Epub ahead of print]

83. Gill SE, Savage K, Wysham WZ, et al. Continuing routine cardiac surveillance in long-term use of pegylated liposomal doxorubicin: is it necessary? Gynecol Oncol. 2013;129(3):544-547.

84. Erriquez J, Olivero M, Mittica G, et al. Xenopatients show the need for precision medicine approach to chemotherapy in ovarian cancer. Oncotarget. 2016;7(18):26181-26191.

85. Butler KA, Hou X, Becker MA, et al. Prevention of Human Lymphoproliferative Tumor Formation in Ovarian Cancer Patient-Derived Xenografts. Neoplasia. 2017:19(8):628-636.

86. Deng C, Zhao J, Zhou S, et al. The Vascular Disrupting Agent CA4P Improves the Antitumor Efficacy of CAR-T Cells in Preclinical Models of Solid Human Tumors. Mol Ther. 2020; 28(1):75-88.

87. Want MY, Konstorum A, Huang RY, et al. Neoantigens retention in patient derived xenograft models mediates autologous $\mathrm{T}$ cells activation in ovarian cancer. Oncoimmunology. 2019;8(6):e1586042.

88. Damelin $M$, Bankovich A Park A, et al. Anti-EFNA4 Calicheamicin Conjugates Effectively Target Triple-Negative Breast and Ovarian Tumor-Initiating Cells to Result in Sustained Tumor Regressions. Clin Cancer Res. 2015;21(18):4165-4173.

89. Cornelison R, Dobbin ZC, Katre AA, et al. Targeting RNA-Polymerase I in Both Chemosensitive and Chemoresistant Populations in Epithelial Ovarian Cancer. Clin Cancer Res. 2017;23(21):6529-6540.

90. Harrington BS, He Y, Davies CM, et al. Cell line and patient-derived xenograft models reveal elevated CDCP1 as a target in high-grade serous ovarian cancer. Br J Cancer. 2016;114(4):417-426.

91. Lee JW, Ryu JY, Yoon G, et al. Sphingosine kinase 1 as a potential therapeutic target in epithelial ovarian cancer. Int J Cancer. 2015;137(1):221-229.

92. Kim HS, Yoon G, Ryu JY, et al. Sphingosine kinase 1 is a reliable prognostic factor and a novel therapeutic target for uterine cervical cancer. Oncotarget. 2015;6(29):26746-26756

93. Choi CH, Ryu JY, Cho YJ, et al. The anti-cancer effects of itraconazole in epithelial ovarian cancer. Sci Rep. 2017;7(1):6552.

94. Pepin D, Sosulski A, Zhang L, et al. AAV9 delivering a modified human Mullerian inhibiting substance as a gene therapy in patient-derived xenografts of ovarian cancer. Proc Natl Acad Sci U S A. 2015;112(32):E4418-4427.

95. Xiao Y, Yu Y, Gao D, et al. Inhibition of CDC25B With WG-391D Impedes the Tumorigenesis of Ovarian Cancer. Front Oncol. 2019;9:236.

96. Ma Y, Wang W, Idowu MO, et al. Ovarian Cancer Relies on Glucose Transporter 1 to Fuel Glycolysis and Growth: Anti-Tumor Activity of BAY-876. Cancers (Basel). 2018;11(1):33.

97. Jiang Y, Lyu T, Che X, Jia N, Li Q, Feng W. Overexpression of SMYD3 in Ovarian Cancer is Associated with Ovarian Cancer Proliferation and Apoptosis via Methylating H3K4 and H4K20. J Cancer. 2019;10(17):4072-4084.

98. Lyu $\mathrm{T}$, Jiang $\mathrm{Y}$, Jia $\mathrm{N}$, et al. SMYD3 promotes implant metastasis of ovarian cancer via H3K4 trimethylation of integrin promoters. Int J Cancer. 2020; 146(6):1553-1567.

99. Kim J, Cho YJ, Ryu JY, et al. CDK7 is a reliable prognostic factor and novel therapeutic target in epithelial ovarian cancer. Gynecol Oncol. 2020; 156(1):211-221.

100. Erriquez J, Becco P, Olivero M, et al. TOP2A gene copy gain predicts response of epithelial ovarian cancers to pegylated liposomal doxorubicin: TOP2A as marker of response to PLD in ovarian cancer. Gynecol Oncol. 2015;138(3):627-633. 